\title{
Descripción de los procesos de elecciones primarias en Estados Unidos
}

\author{
Pablo Amorós González \\ M. Socorro Puy Segura \\ Universidad de Málaga
}

\section{Resumen}

Muchas democracias modernas están mostrando interés en incorporar primarias. Las elecciones primarias son el proceso por el cual el electorado elige a los nominados que se enfrentarán en las elecciones generales. En Estados Unidos hay cuatro tipos de primarias: abiertas, cerradas, semicerradas $y$ top-two. En este artículo describimos cada uno de estos procedimientos. Indicamos en qué escenarios es más conveniente aplicar un tipo u otro de primaria basándonos en los incentivos a votar estratégicamente que generan. Explicamos cómo las primarias favorecen la competencia entre los candidatos a ser nominados, lo que deviene en la elección de mejores nominados.

Palabras clave: votantes registrados, afiliación partidista, elección primaria, votación sincera, votación estratégica.

Clasificación JEL: C72; D72.

\begin{abstract}
Primaries are of key interest in many modern democracies. The primary elections are the process by which the electorate chooses its nominees for general elections. In the US, there are four different types of primary elections: open primaries, close primaries, semi-closed primaries, and top-two primaries. We describe each of these primary procedures. We indicate when it is more convenient to apply one or the other procedure based on the strategic voting incentives generated by each of these primaries. We explain how primary elections foster competition among candidates which translates in the selection of better nominees.
\end{abstract}

Keywords: registered voted, party affiliation, primary elections, sincere voting, strategic voting.

JEL classification: C72, D72.

\section{Introducciónn}

Las elecciones primarias son el proceso por el cual el electorado elige a sus nominados (o a sus líderes) que posteriormente se presentarán a las elecciones generales. El origen de las primarias se remonta al Movimiento Progresista de Estados Unidos, que pretendía introducir más competencia dentro de los partidos políticos a la hora de elegir sus candidatos.

Este movimiento, representado por Robert La Follette, gobernador de Wisconsin desde 1901 hasta 1906, estableció las elecciones primarias por las que los votantes, en vez de los regidores del partido, tenían el derecho de elegir a sus candidatos. 
Antes de esta normativa los candidatos eran elegidos por «caucuses» ${ }^{1}$ privados y en convenciones en vez de por un voto directo de los electores (Hofstadter, 1955; Lovejoy, 1941; Merriam, 1909; Merriam y Overacker, 1928; Ranney, 1975). Tras Wisconsin, otros estados fueron incorporando las elecciones primarias a sus legislaciones.

La evidencia empírica muestra que las elecciones primarias han fomentado la competencia. Eso ocurre especialmente en aquellos estados en los que hay un partido dominante y donde, por tanto, hay escasa competencia entre partidos políticos en las elecciones generales. En estos casos, las elecciones primarias han favorecido la competencia dentro del partido (Key, 1958; Grau, 1981; Jewell y Olson, 1978), lo cual se traduce en la elección de un candidato mejor.

Un candidato es bueno cuando satisface en mayor medida a una gran fracción del electorado. Para ello, o bien el candidato mantiene una ideología cercana a la mediana de la población (cuando esta mediana está bien definida), o bien el candidato es valorado como muy competente por el electorado o ambas. Tal y como señalan Ansolabehere et al. (2006), la competencia en las elecciones primarias mejora el grado de responsabilidad adquirido por los candidatos y mejora el grado en que los candidatos representan al electorado.

Sin embargo, tras más de un siglo de elecciones primarias en Estados Unidos, han surgido algunos síntomas de estancamiento. En este sentido, Ansolabehere et al. $(2006,2010)$ ponen de manifiesto la caída de la competencia en las elecciones primarias. Entre otras razonas señalan al aumento de la ventaja que muestran los candidatos previamente nominados frente a los candidatos recién incorporados y sin experiencia en dicha nominación. Esto se refleja en que se presentan menos candidatos, y en que la primaria tan sólo ratifica al candidato que ya estaba nominado con anterioridad.

A pesar de que hay síntomas de desgaste, pasar de un sistema en el que las élites del partido eligen a los nominados a un sistema en el que hay primarias significa favorecer la competencia entre candidatos. Como muestra Serra (2011), las primarias seleccionan a nominados más competentes y que además corrigen el sesgo ideológico de la cúpula del partido político.

Varios países han mostrado un interés creciente en incorporar los procesos de primarias a sus partidos políticos. En este sentido, Kenig (2009) muestra cómo Dinamarca, Francia, Finlandia, Grecia, Italia, Israel, Japón, Noruega y Reino Unido han incorporado elecciones primarias para elegir a los líderes de alguno de sus partidos políticos. Hazan (1997) también analiza el caso de Israel y Wauters (2010) analiza el caso de Bélgica. Carey y Polga-Hecimovich (2006) muestran cómo algunos países de Latinoamérica están incorporando también procesos de primarias.

A pesar de la reciente expansión de las elecciones primarias fuera de las fronteras de Estados Unidos y del creciente interés que están mostrando algunos partidos polí-

${ }^{1}$ En los Estados Unidos y en Suiza se entiende por caucus («asamblea partidista») el sistema de elegir delegados en varios estados de la Unión, la etapa primaria o preliminar en la que cada partido decide quién recibirá la nominación de su partido a la presidencia. 
ticos por incorporar estos proceso, hay cierto desconocimiento sobre cómo se aplica un proceso de primarias. De hecho, el proceso de primarias no es único en su forma, sino que hay muchas variantes en términos de los votantes con derecho a elegir, el sistema de votación utilizado, e incluso los puestos políticos que son susceptibles de ser elegidos a través de las primarias.

En este artículo vamos a describir los procesos de primarias que se aplican en los distintos estados de Estados Unidos. Indicaremos cómo son los sistemas de votación vigente y qué votantes participan en dichas primarias. Además estudiaremos en qué escenarios es más conveniente aplicar un tipo u otro de primaria basándonos en los incentivos a votar estratégicamente que generan.

El país de referencia es Estados Unidos debido a su larga trayectoria y experiencia en este tipo de elecciones. De hecho, desde sus inicios, estos procesos han sufrido importantes modificaciones, y algunos estados como California o Alaska acaban de aprobar sendas reformas en 2010 y 2011 respectivamente.

El resto del artículo está organizado de la siguiente manera. La Sección 2 describe los distintos sistemas de primarias en EE.UU. La Sección 3 discute los argumentos en contra y a favor de los distintos tipos de elecciones primarias. La Sección 4 presenta las conclusiones.

\section{Tipos de elecciones primarias}

La nominación de candidatos para distintas elecciones en Estados Unidos se realiza a través de caucuses, elecciones primarias y convenciones del partido. Los caucuses son asambleas locales en las que se reúnen los votantes para decidir sus nominados. La votación se realiza a mano alzada. Hay estados que tan solo realizan elecciones primarias, otros que combinan elecciones primarias con caucuses y otros que tan sólo celebran caucuses. Así por ejemplo, los estados de Arizona, Colorado, Delaware y Utah no cuentan con elecciones primarias sino que votan en los caucuses.

En la selección del candidato para las elecciones presidenciales, cada uno de los estados lleva a cabo caucuses o elecciones primarias para elegir a los delegados del partido. Cuando uno de los candidatos cuenta con los votos de suficientes delegados, el partido político celebra su convención para nominar a su candidato para las elecciones. La convención del partido es el acto de nombramiento del nominado, pero la verdadera batalla para la nominación se realiza en los caucuses y en las elecciones primarias de cada uno de los estados.

No solo los nominados para las elecciones presidenciales requieren el apoyo de los votantes vía elecciones primarias o caucuses, sino que también otros muchos cargos tales como nominados para el congreso, senado, gobernador, ayuntamientos o consejo escolar, deben ser elegidos a través de estos procedimientos.

Para participar como votante en las elecciones primarias de EEUU, los ciudadanos estadounidenses deben registrarse (o inscribirse). Para ello hace falta no sólo 
ser ciudadano estadounidense, sino además tener un domicilio o dirección postal en EE.UU. Cada estado tiene su propia normativa sobre cómo registrarse (véase http://www.canivote.org/). Hay una excepción: en el estado de Dakota del Norte los votantes no se registran. Por supuesto, un ciudadano no puede estar registrado en dos estados distintos.

En algunos estados, cuando un ciudadano se registra, existe la opción de afiliarse a un partido político si se desea participar en la elección primaria, en el caucus, o en la convención de dicho partido político. De este modo, y a diferencia de otros países como España, el hecho de estar afiliado a un partido no conlleva una cuota o un coste adicional para el votante, sino una forma de identificarse ideológicamente con un partido.

Un votante, sin embargo, tiene la posibilidad de afiliarse a un partido incluso si al registrarse como votante se identificó como votante independiente. En este caso, y dependiendo del estado, es posible afiliarse a un partido con antelación a la elección primaria de dicho partido o incluso el mismo día de la elección primaria. En ningún caso está permitido estar afiliado simultáneamente a ambos partidos. Un votante también tiene la opción de cambiar su afiliación o de volver a declararse votante independiente.

El grado en que es viable participar como votante en las elecciones primarias y en los caucuses determina la clasificación más utilizada de los tipos de elecciones primarias. Tal y como se describe en (http://www.fairvote.org/), hay cuatro tipos de elecciones primarias: primarias abiertas, primarias cerradas, primarias semicerradas y primarias tipo top-two. Nosotros, adicionalmente clasificamos estos cuatro tipos de elecciones en función de si la primaria es partidista o no. De este modo, describimos los siguientes sistemas de elección de candidatos:

Primarias partidistas: cada partido lleva a cabo de forma separada su elección primaria. Los candidatos de cada uno de los partidos políticos compiten entre sí para ser nominados por dicho partido. Hay distintos tipos:

- primarias abiertas: no importa la afiliación del votante, cualquiera puede votar en la primaria de un partido. La única exclusión es que no es viable votar en las primarias de ambos partidos.

- primarias cerradas: únicamente los votantes registrados con un partido pueden votar en la primaria de dicho partido. En algunos casos los partidos permiten que los votantes independientes (los no afiliados) participen en dicha primaria siempre que se afilien con antelación a la elección primaria.

- primarias semicerradas: los votantes afiliados sólo pueden votar en la primaria de dicho partido, los votantes no afiliados pueden elegir votar en la primaria de uno de los partidos sin que esto requiera afiliarse a dicho partido. 
Primarias no partidistas: hay una única primaria en la que los candidatos, independientemente de su afiliación a un partido político o incluso no afiliados, compiten entre sí para ser nominados. Por tanto, se eliminan las primarias separadas de cada partido político. De momento hay un solo tipo:

- primarias top-two: todos los candidatos se presentan a una única elección. Todos los votantes, afiliados o no, pueden participar en esta elección primaria. Aquellos dos candidatos que obtengan más votos pasan a la elección general.

En la mayoría de los estados, el partido demócrata y el partido republicano utilizan el mismo tipo de primarias. Tal y como se describe en http://www.fairvote.org/, hay varias excepciones que cuentan con un sistema mixto tales como Idaho, Kansas y South Dakota donde las primarias republicanas son cerradas y las demócratas son semicerradas, o Utha donde las republicanas son cerradas y las demócratas son abiertas.

Las primarias tipo top-two han sido una innovación reciente en la política americana. Entre otros casos, este sistema puede dar lugar a que dos candidatos de un mismo partido sean nominados y se enfrenten en las elecciones generales. Estas primarias han sido aprobadas en los estados de Washington en 2004, en California en 2010 y en Alaska en 2011. Hay que mencionar que las primarias top-two han sido recurridas en todos los estados en los que se han implantado. Así por ejemplo, un recurso en el estado de Washington alegaba la inconstitucionalidad de este sistema. El Tribunal Supremo de EE.UU. finalmente falló en contra de dicha inconstitucionalidad. Algunos estados como Arizona, Nueva York y Wisconsin mantienen un debate abierto sobre la conveniencia de modificar su sistema de primarias para incorporar un sistema tipo top-two.

Haciendo un cómputo sobre los sistemas de elecciones primarias en EE.UU., hay un total de 47 estados con unas primarias partidistas, de todas ellas hay 18 estados con primarias cerradas, 18 estados con primarias abiertas, 7 estados con primarias semicerradas y, por último, 4 estados con unas primarias mixtas. Los 4 estados restantes (hasta completar 51) tienen un sistema no partidista, los 3 estados ya mencionados que tienen primarias top-two y Louisiana que tiene un sistema similar al top-two vigente desde 1975.

La diferencia entre el sistema top-two y la primaria en Louisiana es que en este último estado, si tras la votación en la que se presentan todos los candidatos hay uno de ellos que obtiene una mayoría de votos, entonces no se lleva a cabo la segunda ronda entre los dos candidatos más votados. En la primaria top-two siempre se procede a celebrar una segunda ronda de votación entre los dos candidatos más votados. Esta segunda ronda de votación entre los dos candidatos más votados no es una característica única de las primarias tipo top-two. De hecho, las primarias cerradas de los estados de Alabama, Arkansas, Georgia, Mississippi, Carolina del Sur y Texas también celebran una segunda vuelta de votación entre los dos candidatos más votados cuando ninguno de ellos obtiene un determinado porcentaje de votos 
entre los afiliados al partido. La novedad de la primaria tipo top-two no es tanto que conlleve una segunda vuelta de votaciones como que sea una primaria no partidista en la que todos los candidatos, independientemente de su afiliación confluyan en una única elección.

\section{Argumentos a favor y en contra de las distintas primarias}

Antes de valorar las bondades y las carencias asociadas al uso de determinadas elecciones primarias es conveniente definir los escenarios, en términos del reparto histórico de votos entre los partidos, que nos podemos encontrar. De hecho, el uso de un tipo de primaria puede ser más o menos acertado dependiendo de dichos escenarios.

- Estados fuertemente dominados por un partido: uno de los partidos, el demócrata o el republicano, ha ganado históricamente en las últimas elecciones con una amplia mayoría respecto al rival.

- Estados débilmente dominados por un partido: uno de los partidos ha ganado históricamente en las últimas elecciones, pero sin obtener un amplio margen de ventaja respecto a su rival.

- Estados oscilantes entre dos partidos: el margen de victoria demócrata o republicana es muy bajo y, de hecho, ha habido alternancia política entre uno y otro partido en distintas elecciones.

De acuerdo con los resultados electorales de las elecciones presidenciales de los últimos años, los siguientes estados has sido oscilantes entre dos partidos: Colorado, Florida, Iowa, New Hampshire, New Mexico, Nevada, North Carolina, Ohio y Virginia.

Cuando un estado está fuerte o débilmente dominado por un partido, nos referiremos al partido rival como partido dominado.

Esta clasificación está muy determinada por el uso de la regla de la mayoría. El tipo de elecciones que estamos analizando están dirigidas a elegir un candidato: gobernador, senador, alcalde, etcétera. En este contexto, el candidato que obtiene más votos en las elecciones generales (ya sea en un sistema de una sola ronda, o en un sistema de dos rondas) es el ganador y será elegido. Por tanto, el nominado del partido que domina fuertemente un estado, es prácticamente seguro que será elegido en las elecciones generales. Las elecciones primarias son por tanto el filtro, en este contexto, para garantizar la elección de un buen candidato.

Formalizaremos estas consideraciones en un modelo espacial.

Sea $C=\left\{D^{+}, D^{-}, R^{+}, R^{-}\right\}$el grupo de candidatos que optan a ser nominados en las elecciones primarias. La letra $\mathrm{D}$ y $\mathrm{R}$ se refieren a los candidatos demócratas y republicanos respectivamente. Además, los símbolos + y - significan extremistas y moderados respectivamente. Por ejemplo, $\mathrm{D}^{+}$se refiere a un candidato demócrata 
extremista y $\mathrm{R}^{-}$se refiere a un candidato republicano moderado. Podemos identificar a cada candidato con una posición en un espacio político unidimensional, el intervalo unitario $[0,1]$, que refleje su ideología (izquierda-derecha o liberal-conservador). De esta manera, podemos ordenar el conjunto $\mathrm{C}$ de forma que $\mathrm{D}^{+}<\mathrm{D}^{-}<\mathrm{R}^{-}<\mathrm{R}^{+}$.

\section{GRÁFICO 1}

\section{LOCALIZACIÓN DE LOS CANDIDATOS A SER NOMINADOS}

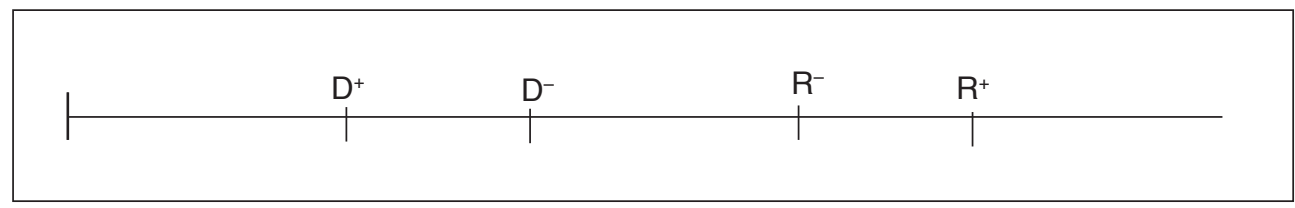

Sea $\pi_{i} \in[0,1]$ la política ideal de un votante. Las preferencias de los votantes sobre los candidatos a elecciones primarias vienen representadas por la distancia entre la política ideal y la posición del candidato, además de por el carisma o competencia del candidato. Sea $x \in C$,

$$
u_{i}(x)=v_{x}-\left|x-\pi_{i}\right|
$$

donde $v_{x}>0$ mide el carisma o competencia del candidato $x$. Si la diferencia de carisma entre candidatos no es muy grande, entonces el carisma apenas distorsiona las preferencias basadas sólo en distancia al candidato.

Nos referimos al mediano poblacional como aquel votante cuya política ideal es la mediana de la distribución de políticas ideales, y denotamos su política ideal como $\pi_{m} \in[0,1]$. Para facilitar el análisis, consideramos que las preferencias agregadas de acuerdo con la regla de la mayoría coinciden con las preferencias del votante mediano. En el caso de no considerar el parámetro que mide el carisma del candidato, esta condición se satisface directamente ya que las preferencias serían de un solo pico de acuerdo con el orden de los candidatos $\left(\mathrm{D}^{+}<\mathrm{D}^{-}<\mathrm{R}^{-}<\mathrm{R}^{+}\right)$. Sin embargo, diferencias notables en el carisma o competencia de los candidatos pueden generar preferencias que no sean de un solo pico respecto a dicho orden.

Formalmente, definimos que un estado está fuertemente dominado por el partido $\mathrm{D}$ cuando se cumplen las siguientes dos condiciones: (i) el candidato $\mathrm{D}^{+}$es más preferido para el mediano poblacional que $\mathrm{R}^{-}$y $\mathrm{R}^{+}$; (ii) el candidato $\mathrm{D}^{-}$es más preferido para el mediano poblacional que $\mathrm{R}^{-}$y $\mathrm{R}^{+}$.

La condición (i) implica que

$$
\pi_{m} \leq v_{D^{+} R^{-}}+\frac{D^{+}+R^{-}}{2} ; \pi_{m} \leq v_{D^{-} R^{+}}+\frac{D^{-}+R^{+}}{2}
$$


donde $v_{D^{+} R^{-}}=v_{D^{+}}-v_{R^{-}}$mide la diferencia de carisma entre el candidato $\mathrm{D}^{+} \mathrm{y}$ el candidato $\mathrm{R}^{-}$y por tanto, $v_{D^{+} R^{+}}=v_{D^{+}}-v_{R^{+}}$.

La condición (ii) implica que

$$
\pi_{m} \leq v_{D^{-} R^{-}}+\frac{D^{-}+R^{-}}{2} ; \pi_{m} \leq v_{D^{-R^{+}}}+\frac{D^{-}+R^{+}}{2}
$$

Decimos que un estado está débilmente dominado por el partido D cuando solo se cumple una de las condiciones anteriores: (i) o (ii). Esto implica que hay algún candidato del partido $\mathrm{R}$ que es más preferido para el mediano que alguno de los candidatos del partido D. Por ejemplo, puede ocurrir que el candidato $\mathrm{D}^{+}$sea menos preferido para el mediano que el candidato $\mathrm{R}^{-}$(es decir, no se cumple $\mathrm{i}$ ), pero que el candidato $\mathrm{D}^{-}$sea más preferido para el mediano que los candidatos $\mathrm{R}^{-}$y $\mathrm{R}^{+}$(es decir, sí se cumple ii).

Por último, un estado es oscilante cuando ningún partido domina fuertemente o débilmente en dicho estado.

A continuación analizamos cada una de las elecciones primarias.

\subsection{Primarias cerradas}

En una primaria cerrada sólo votan aquellos ciudadanos que están registrados y afiliados a dicho partido. En términos de los incentivos que tienen estos votantes a la hora de elegir un nominado podemos pensar en los siguientes comportamientos:

- Voto sincero. El votante opta por su candidato más preferido valorando tanto su cercanía ideológica como su carisma o competencia.

- Voto estratégico. En este caso, el votante opta por aquel candidato que tenga más opciones de ganar al nominado del partido rival. Un votante, entonces, puede primar su voto hacia un candidato más centrista, o hacia otro que es considerado como más competente o más carismático siempre que dicho candidato tenga más opciones de ganar las elecciones generales.

Aunque esta descripción de comportamientos no es exhaustiva, es un buen reflejo de cómo votan la mayoría de los ciudadanos en este tipo de primarias. Es relevante distinguir cuándo nos encontraremos con un tipo u otro de comportamiento y cuáles serán las consecuencias.

El hecho de que un votante sea sincero o estratégico dependerá básicamente de cuál sea la posición del partido en dicho estado. Así por ejemplo, si el partido político que celebra la elección primaria domina fuertemente un estado, entonces nos encontraremos con voto sincero. De hecho, es una estrategia (débilmente) dominante para los afiliados a dicho partido votar por su candidato más preferido.

Si por el contrario el estado oscila entre dos partidos o un partido domina débilmente, los votantes, anticipando el resultado de las elecciones generales, tienen 
incentivo para votar estratégicamente. En estos escenarios, los votantes estarán más preocupados por ganar las elecciones generales que por votar a su candidato más preferido.

El mayor problema de las primarias cerradas se debe, por tanto, al voto sincero cuando un estado está fuertemente dominado por un partido. En este caso, los votantes saben que el candidato nominado en las primarias del partido dominante ganará las elecciones generales.

De acuerdo con la definición de partido fuertemente dominante, tanto $\mathrm{D}^{+}$como $\mathrm{D}^{-}$son preferidos por el mediano poblacional a los candidatos $\mathrm{R}^{+} \mathrm{y} \mathrm{R}^{-}$. Gráficamente, si $\pi_{\mathrm{m}}^{\mathrm{D}}$ es el mediano de los votantes registrados con el partido $\mathrm{D}, \mathrm{y} \pi_{\mathrm{m}}$ es el mediano poblacional, entonces hay dos posibles escenarios que mostramos en el Gráfico 2. En el gráfico superior el mediano del partido prefiere $\mathrm{D}^{+}$frente a $\mathrm{D}^{-}$, tal y como indican sus preferencias con pico en $\pi_{\mathrm{m}}^{\mathrm{D}}$. En el gráfico inferior, el mediano del partido prefiere $\mathrm{D}^{-}$frente $\mathrm{D}^{+}$. Por tanto, tal y como ocurre en el gráfico superior, el nominado del partido estrictamente dominante puede ser demasiado radical ya que dicho nominado es elegido en la primaria cerrada. Incluso siendo radical, dicho nominado gana a ambos candidatos del partido rival. El votante mediano de la población, sin embargo, preferiría al candidato $\mathrm{D}^{-}$frente al candidato $\mathrm{D}^{+}$. Este problema no tiene una clara solución a menos que el estado opte por una primaria abierta o se lleve a cabo una primaria no partidista.

\section{GRÁFICO 2}

LOCALIZACIÓN DEL MEDIANO DEL PARTIDO Y EL MEDIANO POBLACIONAL

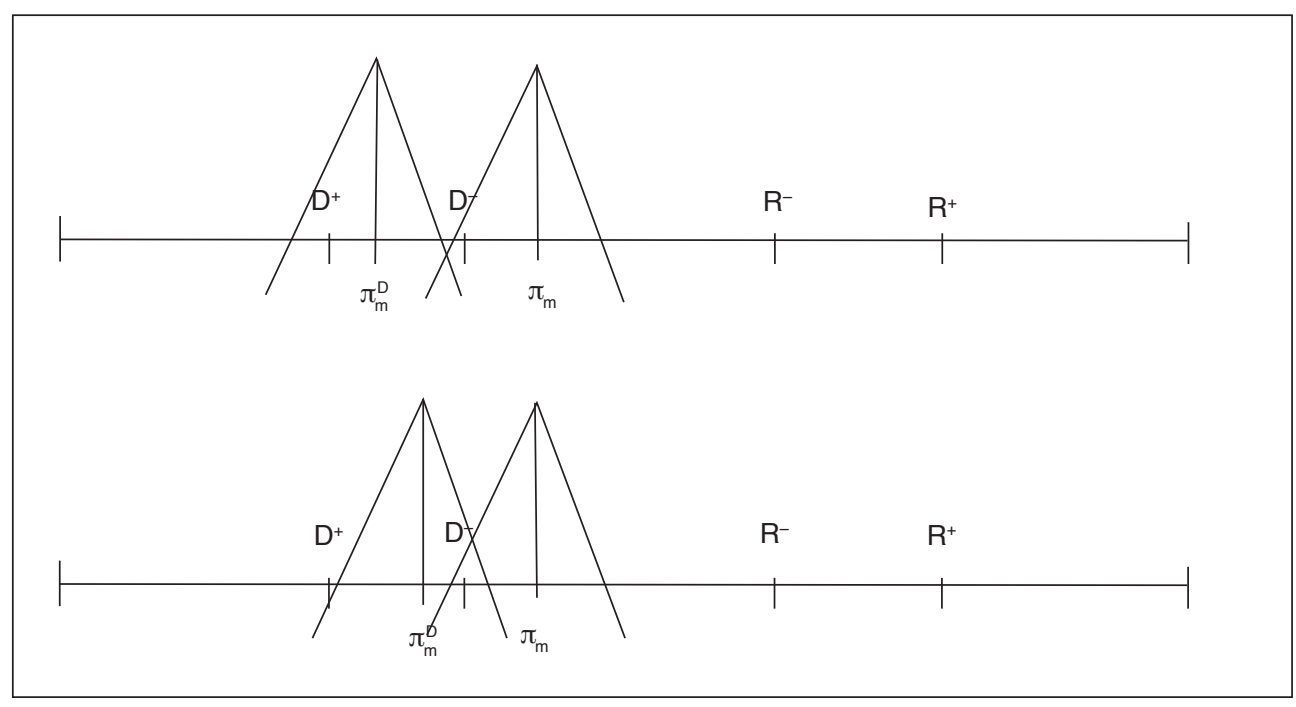


Cuando el voto es estratégico, cada votante tiene en cuenta que el candidato al que vota se enfrentará a continuación al nominado del partido rival. Si el estado no está claramente dominado por un partido, el votante tendrá en cuenta que un candidato demasiado extremista, en términos ideológicos, no será el adecuado para ganar las elecciones generales. De acuerdo con el modelo presentado, cuando un partido domina débilmente en un estado, o bien el candidato $\mathrm{D}^{-}$o bien el candidato $\mathrm{D}^{+}$es preferido por el mediano poblacional al candidato $\mathrm{R}^{-} \mathrm{y} \mathrm{R}^{+}$. Sin embargo, los votantes anticiparán el resultado de las elecciones generales y optarán por votar al candidato con más opciones incluso en una situación en que otro candidato sea más preferido. Algo similar ocurre si el estado es oscilante. Votando a $\mathrm{D}^{-} \mathrm{o}$ a $\mathrm{R}^{-}$el partido tiene alguna opción de ganar, y dicha opción se elimina cuando se vota por el candidato más extremista.

Los propios votantes en esta situación buscan un candidato que satisfaga al votante mediano de la población y evitarán a aquellos candidatos con pocas opciones de ganar las elecciones generales. Los candidatos de más consenso, con ideología más centrista o con más competencia, tendrán más opciones de ser nominados.

\subsection{Primarias abiertas}

En una primaria abierta cualquier votante, independientemente de su afiliación, puede participar en una y sólo una de las primarias. Nos encontramos con que un votante afiliado a un partido puede votar en las primarias de su rival. En este caso hay dos decisiones consecutivas, la primera consiste en optar por la primaria de un partido y la segunda consiste en decidir por qué candidato se vota.

Por tanto, podemos tener una decisión sincera o estratégica en cuanto a la selección de partido y una decisión sincera o estratégica en cuanto a la votación del candidato. Distinguimos cuatro tipos de estrategias:

- Elección sincera del partido y voto sincero: el votante participa en la primaria del partido que prefiere y vota de forma sincera por su candidato más preferido.

- Elección sincera del partido y voto estratégico: el votante participa en la primaria del partido que prefiere y vota por aquel candidato que tenga más opciones de ganar las elecciones generales.

- Elección estratégica del partido y voto sincero: el votante participa en la primaria del partido rival y opta por aquel candidato del partido rival que le resulta más preferido.

- Elección estratégica del partido y voto estratégico: el votante participa en la primaria del partido rival y opta por aquel candidato que tenga menos opciones de ganar las elecciones generales. De este modo, favorece indirectamente a su candidato más preferido. 
Vemos que las primarias abiertas permiten no solo llevar a cabo las estrategias que ya estaban disponibles en las primarias cerradas, sino que además permiten votar en las primarias del otro partido. Como hemos mencionado antes, cuando un votante puede votar en las primarias del partido que no es su primer partido, se pueden corregir algunas de las deficiencias de las primarias cerradas. En particular, si en un estado domina fuertemente un partido, los afiliados al partido rival o los votantes independientes pueden participar en la primaria del partido dominante y de este modo moderar la votación para que el nominado sea un candidato de más consenso, es decir, un candidato más centrista o incluso más competente o carismático.

Aparecen también otros efectos no tan positivos. En un estado oscilante entre dos partidos o donde domina débilmente un partido, hay incentivos para que un votante sea estratégico en la elección de partido con el fin de votar por un candidato con menos posibilidades de ganar en las elecciones generales. De este modo, puede aumentar el margen de victoria de su primer partido, o incluso favorecer que su partido gane en el caso de que la elección general estuviera muy reñida. Este es, por tanto, un efecto perverso de las primarias abiertas que no se puede evitar a menos que las primarias vuelvan a ser cerradas o semicerradas.

\subsection{Primarias semicerradas}

Los afiliados a un partido y cualquier votante independiente pueden participar en la primaria de dicho partido. Los votantes afiliados al partido sólo tienen dos opciones, votar de forma sincera o votar de forma estratégica, exactamente igual que en el caso de una primaria cerrada. Los votantes independientes, sin embargo, tienen las cuatro opciones descritas en el caso de unas primarias abiertas.

Cuando el estado está fuertemente dominado por un partido, la primaria semicerrada permite que los votantes independientes participen en la elección del nominado del partido dominante. Esto contribuirá a la elección de un nominado más centrista, pero en este caso, una primaria abierta tendría un efecto más potente en este sentido.

Cuando el estado oscila entre dos partidos o está débilmente dominado por un partido, la primaria semicerrada evita el efecto perverso de votar al candidato débil del partido rival. Esto se debe a que los únicos votantes con derecho a elegir partido son los no afiliados que se declaran independientes. Estos votantes, por tanto, no tienen una clara preferencia por un partido o por una ideología. Si deciden votar en una primaria sería meramente porque tienen una preferencia por determinado candidato, porque les parece más moderado o más competente. De este modo, la participación en las primarias de los votantes independientes puede favorecer la elección de un candidato mejor. Adicionalmente, si en uno de los partidos hay un candidato que tiene claras opciones de ser nominado, el votante independiente puede optar por participar en las primarias del otro partido para que su voto cuente. 
Nuevamente, en este caso, optará por el candidato que considere más moderado o capaz.

\subsection{Primarias top-two}

Todos los votantes participan en una única elección primaria. Los dos candidatos con más votos son nominados y se enfrentarán en las elecciones generales. En términos de los incentivos que tienen estos votantes a la hora de elegir un nominado distinguimos dos comportamientos:

- Voto sincero: votar en la primaria por el candidato más preferido teniendo en cuenta la cercanía ideológica y su competencia.

- Voto estratégico: votar en la primaria por un candidato que no sea el más preferido o el más competente con el fin de evitar que uno de los candidatos menos preferidos acabe ganando las elecciones generales.

La predicción sobre el comportamiento de los votantes no es nada obvia. Los votantes tienen dos rondas de votación y un votante tiene la opción de usar distintos tipos de voto estratégico. En un artículo reciente, Amorós y Puy (2013) formalizan, utilizando la Teoría de Juegos, la situación estratégica de los candidatos y votantes en las primarias top-two.

En un estado fuertemente dominado por un partido es de esperar que dos candidatos pertenecientes o afiliados a un mismo partido acaben siendo nominados a las elecciones generales. Las elecciones generales serán más competitivas, lo cual a su vez incentivará la participación electoral. Intuitivamente esperaríamos que, al igual que en el caso de una primaria abierta, (incluso con más intensidad en este caso debido a la mayor participación) se contribuyera a elegir un candidato más moderado o más competente en comparación con el elegido en una primaria cerrada. Sin embargo, tal y como muestran Amorós y Puy (2013), cualquier candidato nominado en una primaria cerrada también puede ser elegido en las primarias top-two. Es cierto que la primaria top-two puede elegir candidatos más moderados, pero no puede evitar la elección de candidatos extremistas.

En un estado oscilante o débilmente dominado por un partido y de acuerdo con los resultados en Amorós y Puy (2013), las primarias top-two apenas contribuyen a la elección de un candidato más moderado ya que no evitan la elección de un candidato extremista. Lo que sí permite (y esto lo hace con más intensidad que las primarias cerradas), es que haya más alternancia política entre los dos partidos cuando el estado no está fuertemente dominado por un partido.

Por otro lado, la mayor novedad de este tipo de primarias es la eliminación del partido político como institución indispensable en la selección de nominados. En 
este sentido, este tipo de primaria transfiere esa competencia a todo el electorado, cualquiera que sea su condición de afiliado o independiente.

\section{Conclusiones}

En este artículo hemos descrito cómo funcionan las elecciones primarias en Estados Unidos. Esta es una realidad en la que merece la pena profundizar dado que muchos países (entre ellos España), están mostrando un interés creciente en mejorar la calidad de las reglas y procesos electorales. La democracia se materializa en reglas concretas de votación. Hay que estudiar y analizar con rigor dichas reglas para garantizar el pleno funcionamiento de una democracia.

Los procesos de primarias son cruciales para elegir buenos candidatos. Al igual que la competencia entre empresas contribuye a que las empresas innoven, la competencia en el proceso de nominación de un candidato contribuye a la creación de nuevos discursos y a la propuesta de medidas políticas más convincentes para el electorado. Conforme se amplía el electorado con voto en el proceso de primarias, los candidatos a ser nominados requieren más preparación. Desde este punto de vista, todos los procesos de primarias presentados son adecuados para fomentar la competencia entre candidatos Todos ellos permiten, en mayor o menor medida, que un gran grupo de ciudadanos participen en el proceso de elección de nominados.

Comparando estos sistemas, y teniendo en cuenta que la peor primaria es no tener primaria, hemos argumentado que las primarias abiertas son más adecuadas en aquellos estados que están fuertemente dominados por un partido. En este caso, el nominado del partido dominante gana las elecciones generales. Para que este nominado represente mejor las preferencias de todo el electorado habría que promover unas primarias abiertas.

Las primarias abiertas no son adecuadas cuando el estado está débilmente dominado o bien oscila entre dos partidos. Esto es debido a que permiten, de forma perversa, que los votantes de un partido elijan un rival débil para favorecer la victoria de su partido en las elecciones generales. Tanto una primaria cerrada como semicerrada evitan este efecto perverso. Por tanto, sugerimos que una primaria cerrada o semicerrada es más adecuada cuando no hay un partido dominante en dicho estado.

La última novedad en la democracia americana ha sido la incorporación de las primarias top-two que prescinden del partido político para llevar a cabo la nominación de candidatos. Este nuevo sistema está motivado por la búsqueda de más competencia entre candidatos y el deseo de que los candidatos estén incentivados por satisfacer al electorado y no a la cúpula del partido político. Las primarias tipo top-two no evitan resultados en los que un candidato extremista es elegido, pero sin embargo, y a diferencia de lo que ocurre en una primaria cerrada, los candidatos más moderados tienen más opciones de ser nominados. La principal virtud de las prima- 
rias top-two es ser no partidista y de este modo es adecuada en esos escenarios en los que los incentivos de un partido político no están en consonancia con los intereses de los ciudadanos.

Finalmente, destacamos que desde un punto de vista empírico Ansolabehere et al. (2010) muestran que la introducción de elecciones primarias no ha generado posiciones más extremistas, tal y como podíamos esperar cuando las primarias son cerradas. Al contrario, las primarias han generado presión para que se produzca convergencia en ideologías y moderación.

\section{Referencias bibliográficas}

[1] AMORÓS, P. y PUY, M. S. (2013): «The Closed Primaries versus the Top-two Primary», mimeo, Universidad de Málaga.

[2] ANSOLABEHERE, S.; HANSEN, J. M.; Hirano, S. y SNYDER, J. M. (2006): «The Decline of Competition in US Primary Elections, 1908-2004», The Marketplace of Democracy: Electoral Competition and American Politics, pp. 74-101.

[3] ANSOLABEHERE, S.; HANSEN, J. M.; HIRANO, S. y SNYDER, J. M. (2010): «More Democracy: The Direct Primary and Competition in U.S. Elections», Studies in American Political Development, 24, pp. 190-205.

[4] ANSOLABEHERE, S.; HANSEN, J. M.; HIRANO, S. y SNYDER, J. M. (2010): «Primary Elections and Partisan Polarization in the U.S. Congress», Quarterly Journal of Political Science, 5, pp. 169-191.

[5] CAREY, J. M. y POLGA-HECIMOVICH, J. (2006): «Primary Elections and Candidate Strength in Latin America», Journal of Politics, 68, pp. 530-543.

[6] GRAU, G. H. (1981): «Competition in State Legislative Primaries», Legislative Studies Quarterly, 6, pp. 35-54.

[7] JEWELL, M. E. y OLSON, D. M. (1978): American State Political Parties and Elections. Homewood, Ill.: Dorsey.

[8] HAZAN, R. Y. (1997): «The 1996 Intra-Party Elections in Israel: Adopting Party Primaries», Electoral Studies, 16, pp. 95-103.

[9] HOFSTADTER, R. (1955): The age of reform: from Bryan to F.D.R., New York : Vintage Books.

[10] KENIG, O. (2009): «Democratization of party leadership selection: do wider electorates produce more competitive contests?", Electoral Studies, 28, pp. 240-247.

[11] KEY, V. O. (1958): Politics, Parties, and Pressure Groups, 4th. Edition. New York: Thomas Y. Cromwell.

[12] LOVEJOY, A. F. (1941): La Follette and the establishment of the direct primary in Wisconsin: 1890-1904, Yale University Press.

[13] MERRIAM, C. E. (1909): Primary Elections: a study of the history and tendencies of primary election legislation. University of Chicago Press.

[14] MERRIAM, C. E. y OVERACKER, L. (1928): Primary elections, University of Chicago Press.

[15] RANNEY, A. (1975): Curing the Mischiefs of Faction: Party Reform in America, University of California Press. 
[16] SERRA, G. (2011): «Why primaries? The party's trade-off between policy and valence», Journal of Theoretical Politics, 23, pp. 21-51.

[17] WAUTERS, B. (2010): «Explaining Participation in Intra-Party Elections. Evidence from Belgian Political Parties», Party Politics, 16, pp. 237-259. 